\title{
Relation between FQHE Plateau Width and Valley Energy
}

\author{
Shosuke Sasaki \\ Center for Advanced High Magnetic Field Science, Graduate School of Science, Osaka University, \\ Osaka, Japan \\ Email: sasaki@mag.ahmf.sci.osaka-u.ac.jp, zazensou@gmail.com
}

Received 23 May 2015; accepted 27 June 2015; published 30 June 2015

Copyright (c) 2015 by author and Scientific Research Publishing Inc.

This work is licensed under the Creative Commons Attribution International License (CC BY).

http://creativecommons.org/licenses/by/4.0/

(c) † Open Access

\begin{abstract}
We have investigated the Fractional Quantum Hall Effect (FQHE) on the fundamental Hamiltonian with the Coulomb interactions between normal electrons without any quasi particle. The electron pairs placed in the Landau orbitals can transfer to many empty orbitals. The number of the quantum transitions decreases discontinuously when the filling factor $v$ deviates from the specific fractional number of $v_{0}$. The discontinuous decreasing produces the energy valley at the specific filling factors $v_{0}=2 / 3,4 / 5,3 / 5,4 / 7,3 / 7,2 / 5,1 / 3$ and so on. The diagonal elements of the total Hamiltonian and the number of the quantum transitions give the total energy of the FQH states. The energy per electron has the discontinuous spectrum depending on the filling factor $v$. We obtain the function form of the energy per electron in the quantum Hall system. Then the theoretical Hall resistance curve is calculated near several filling factors. Therein the quantum Hall plateaus are derived from the energy valleys. The depths of the energy valleys are compared with the widths of the quantum Hall plateaus appearing in the experimental data of the Hall resistance. Our theoretical results are in good agreement with the experimental results.
\end{abstract}

\section{Keywords}

Fractional Quantum Hall Effect, 2D Electron System, Quantum Theory, Hall Resistance

\section{Introduction}

The quantum Hall effect is derived by the total Hamiltonian $H_{\mathrm{T}}$ of a many-electron system which is composed of the single electron Hamiltonian $H_{0, i}$ of the i-th electron and the Coulomb interaction between electrons as follows: 


$$
\begin{aligned}
& H_{\mathrm{T}}=\sum_{i=1}^{N} H_{0, i}+\sum_{i=1}^{N-1} \sum_{j>i}^{N} \frac{e^{2}}{4 \pi \varepsilon \sqrt{\left(x_{i}-x_{j}\right)^{2}+\left(y_{i}-y_{j}\right)^{2}+\left(z_{i}-z_{j}\right)^{2}}} \\
& H_{0, i}=\frac{\left(\boldsymbol{p}_{i}+e \boldsymbol{A}\right)^{2}}{2 m^{*}}+U\left(y_{i}\right)+V_{\text {Thin }}\left(z_{i}\right)+\mu_{\mathrm{B}} g^{*} s_{i, z} B
\end{aligned}
$$

where $m^{*}, e, \boldsymbol{p}_{i}$ and $N$ are the effective mass, the elementary charge, the momentum and the total number of electrons. Therein $V_{\text {Thin }}(z)$ indicates the potential of the $z$-direction which confines the electrons to an ultrathin conducting layer. Also $U(y)$ is the electric potential along the Hall voltage (y-direction). The vector potential, $\boldsymbol{A}$, has the components,

$$
\boldsymbol{A}=(-y B, 0,0),
$$

where $B$ is the strength of the magnetic field. The last term of Equation (2) indicates the Zeeman energy where $g^{*}$ is the effective g-factor, $\mu_{\mathrm{B}}$ is the Bohr magneton $\left(\mu_{\mathrm{B}}=\hbar e /(2 m)\right)$ and $s_{i, z}$ is the $z$-component of the $i$-th electron spin operator. The details have been explained in the previous papers [1]-[14]. When the Coulomb interaction between electrons is ignored in the quasi-2D electron system, the Hamiltonian of the single electron is exactly diagonalized same as in the Landau solution. At a filling factor $v<1$, all the electrons are placed in the Landau orbitals with the Landau level number $L=0$. The residual Landau orbitals ( $L=0)$ are empty. So there are various electron-configurations in the Landau orbitals. We divide the total Hamiltonian $H_{\mathrm{T}}$ into the diagonal part $H_{\mathrm{D}}$ and the non-diagonal part $H_{\mathrm{I}}$ as follows;

$$
H_{\mathrm{T}}=H_{\mathrm{D}}+H_{\mathrm{I}}
$$

We define two symbols $W$ and $C$ which are the expectation values of $H_{\mathrm{T}}$ and the Coulomb energy, respectively. We call $C$ "classical Coulomb energy" (the expectation value of the Coulomb interaction). Then the classical Coulomb energy becomes a minimum for only one electron configuration in the Landau orbitals. This property has been proven in the previous paper [9]. That is to say $W$ becomes a minimum at the only one electron configuration. The electron configuration gives the single ground state for each value of $v$. The residual Coulomb interaction $H_{\mathrm{I}}$ (non diagonal part of $H_{\mathrm{T}}$ ) produces many quantum transitions from the ground state. We have examined the perturbation energy via these quantum transitions in details in the previous papers [1]-[14]. Then all the electron pairs placed in the nearest Landau orbitals can transfer to all the empty orbitals at the specific filling factors $v_{0}$. When the filling factor $v$ deviates from the fractional numbers $v_{0}$ the number of the transitions abruptly decreases. The reason comes from the combined effects of the momentum conservation along the $\mathrm{x}$ direction (current direction), the most uniform electron configuration and the Pauli exclusion-principle. The abrupt decreasing of the transition number yields the valley structure in the perturbation energy. That is to say the energy $\chi(v)$ of the nearest electron pair takes a minimum at $v=v_{0}$ and the energy $\chi(v)$ for $v \neq v_{0}$ is higher than $\chi\left(v_{0}\right)$. Then $\lim _{v \rightarrow v_{0} \pm \varepsilon} \chi(v)-\chi\left(v_{0}\right)$ gives the energy gap (valley depth) as proven in the previous papers [9] [12] [13].

The total energy of the quantum Hall system is the sum of $W$ (expectation value of $H_{\mathrm{T}}$ ) and all the pair energy of electrons (placed in nearest orbital pairs and more distant orbital pairs), because the Coulomb interaction works between two electrons. We will study the function form of the expectation value $W$ in the next section. Then we get the energy spectrum of the quantum Hall system which is quite different from the Halperin result [15]. The valley depth in the pair energy and the function form of $W$ give the quantum Hall plateaus at the specific filling factors $v_{0}$. The theoretical results are in good agreement with the experimental data.

\section{Expectation Value of the Total Hamiltonian and Its $v$-Dependence}

We describe the expectation value of the total Hamiltonian $H_{\mathrm{T}}$ by the symbol $W$ which is the sum of the single electron energies and the expectation value of the classical Coulomb energy as follows;

$$
W\left(p_{1}, \cdots, p_{N}\right)=\sum_{i=1}^{N} E_{0}\left(p_{i}\right)+C\left(p_{1}, \cdots, p_{N}\right)
$$

where $E_{0}\left(p_{i}\right)$ is the single electron energy of the $i$-th electron and $C\left(p_{1}, \cdots, p_{N}\right)$ is the expectation value of 
the Coulomb interaction between electrons. The total number of Landau states with $L=0$ is equal to $e B \ell d / 2 \pi \hbar$ where $\ell$ and $d$ are the length and the width of a quantum Hall device respectively. The total charge of electrons at the filling factor $v$ is the product of $v \times(-e)$ and the total number of Landau states. The total charge is divided by the area $\ell d$, and then we obtain the charge density as

$$
\text { macroscopic charge density } \sigma=-\frac{e^{2} B}{2 \pi \hbar} v
$$

Figure 1 shows one of the experimental data [16]. The upper figure indicates the Hall resistance $R_{\mathrm{H}}$ divided by the Klitzing constant $R_{\mathrm{K}}=2 \pi \hbar / e^{2}$. The value of $R_{\mathrm{H}} / R_{\mathrm{K}}$ is equal to $1 / v$ which is almost proportional to the magnetic field strength $B$ except the Hall plateau regions. This property is easily seen by comparing the data with the red line. That is to say, $B v$ is nearly equal to the constant value. Equation (5) means the charge density $\sigma$ to be proportional to $B v$. Accordingly the macroscopic Coulomb energy $C_{\text {Macroscopic }}(\sigma)$ may be treated to be a constant value in the experiment of Figure 1.

We next examine the microscopic charge-distribution in more details. Figure 2 shows the electron configuration with a minimum classical-Coulomb-energy at $v=2 / 3$. Therein the bold lines express the occupied orbitals with electron and the dashed lines indicate the empty orbitals. The electron pair located at the orbitals $\mathrm{AB}$ is one example of the nearest-electron-pairs. Two electrons placed at B and C show the second nearest pair. The electron pair at $A$ and $C$ is the third nearest pair. The pair at $A$ and $D$ is the fourth nearest pair and so on. The classical Coulomb energy between two electrons is expressed by the symbols $\xi, \eta, \varsigma$ and $\kappa$ respectively as in Figure 2. Therein $\xi$ is the largest one of the classical Coulomb pair energy, $\eta$ is the second largest, $\varsigma$ is the third largest, $\kappa$ is the fourth largest and so on.

The classical Coulomb energy between the pair (A, C) is weakened by the screening (shielding) effect of electron B. Also the classical Coulomb energy between the pair (A, D) is weakened by the screening effect of electrons B and C. Accordingly the $v$-dependence of the classical Coulomb energy mainly comes from the first nearest and the second nearest pairs. The number of the more distant pairs (third, fourth, fifth and so on) are enormous many. The total number of electron pairs is $N(N-1) / 2$. On the other hand the total number of the first and second nearest pairs is $N$. Accordingly the residual energies (namely the sum of all the more distant pair

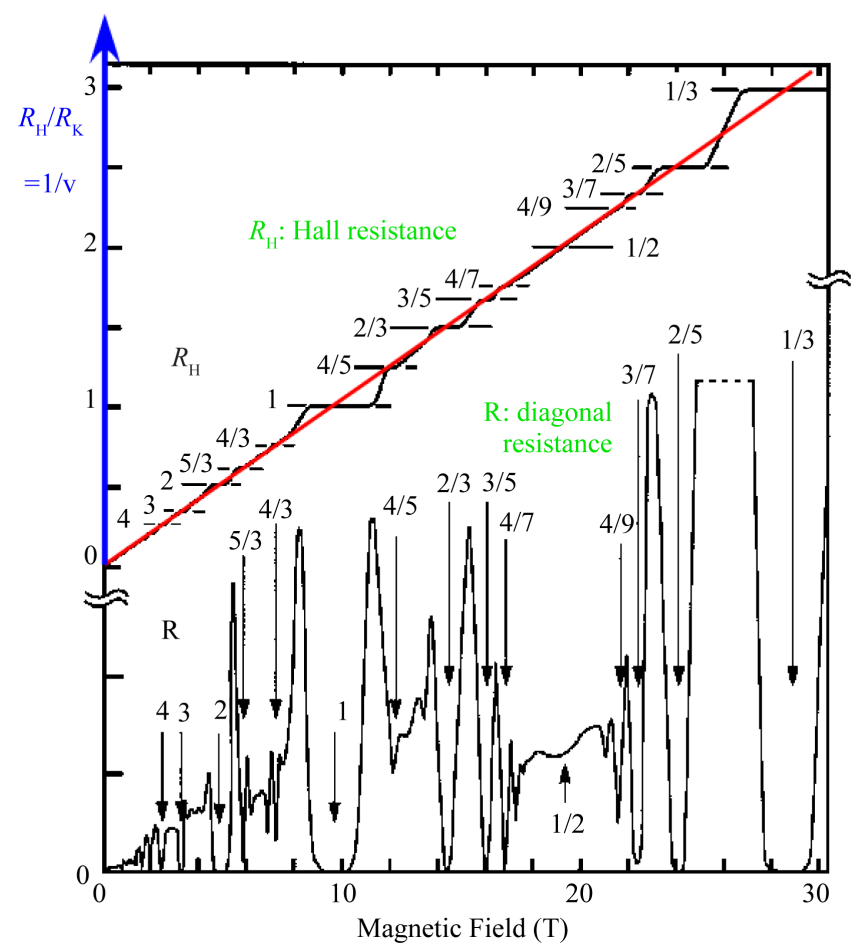

Figure 1. Hall resistance $R_{\mathrm{H}}$ and diagonal resistance $R$ in ultrahighmobility device in [16]. 


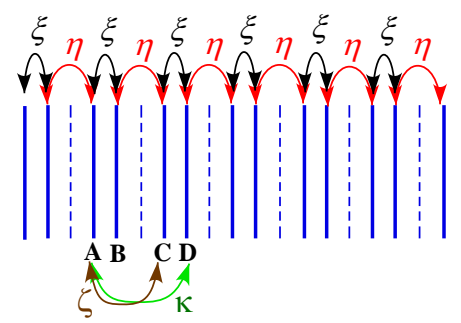

Figure 2. Electron configuration with the minimum classical Coulomb energy at $v=2 / 3$.

energies) may be approximated by the macroscopic Coulomb energy $C_{\text {Macroscopic }}(\sigma)$ which is a constant value mentioned above. On the other hand the sum of the first and second nearest pair-energies is strongly dependent upon the filling factor. The $v$-dependence is examined for various filling factors as follows:

(Case of $v=2 / 3$ )

We can ignore the boundary effect in both ends for a macroscopic electron-number $N$. Then the number of the first nearest pairs is equal to $N / 2$ and the number of the second nearest pairs is equal to $N / 2$ for the configuration of Figure 2. Then the sum of the classical Coulomb energies between the first nearest pairs is equal to $\xi \cdot N / 2$ and that between the second nearest pairs is equal to $\eta \cdot N / 2$. Accordingly, the total classical Coulomb energy $C\left(p_{1}, \cdots, p_{N}\right)$ is approximated by

$$
C\left(p_{1}, \cdots, p_{N}\right) \approx[(\xi / 2)+(\eta / 2)] N+C_{\text {Macroscopic }}(\sigma) \text { at } v=2 / 3
$$

Next we estimate the classical Coulomb energy for $v=3 / 5,4 / 7$, 5/7 where the electron-configurations with the minimum classical Coulomb energy are shown in Figures 3-5.

(Case of $v=3 / 5$ ) The number of the first nearest pairs is equal to $(1 / 3) N$ and the number of the second nearest pairs is equal to $(2 / 3) N$ at $v=3 / 5$ as seen in Figure 3 . Then the total classical Coulomb energy is nearly equal to

$$
C\left(p_{1}, \cdots, p_{N}\right) \approx[(\xi / 3)+(2 \eta / 3)] N+C_{\text {Macroscopic }}(\sigma) \text { at } v=3 / 5
$$

(Case of $v=4 / 7$ ) Figure 4 shows that the number of the first nearest pairs is equal to $(1 / 4) N$ and the number of the second nearest pairs is equal to $(3 / 4) N$ at $v=4 / 7$. The total classical Coulomb energy is given by

$$
C\left(p_{1}, \cdots, p_{N}\right) \approx[(\xi / 4)+(3 \eta / 4)] N+C_{\text {Macroscopic }}(\sigma) \text { for } v=4 / 7
$$

(Case of $v=5 / 7)$ The number of the first nearest pairs is equal to $(3 / 5) N$ and the number of the second nearest pairs is equal to $(2 / 5) N$ at $v=5 / 7$ as easily seen in Figure 5. Then the total classical Coulomb energy is

$$
C\left(p_{1}, \cdots, p_{N}\right) \approx[(3 \xi / 5)+(2 \eta / 5)] N+C_{\text {Macroscopic }}(\sigma) \text { at } v=5 / 7
$$

(Any case of $1 / 2<v<1$ )

We calculate the classical Coulomb energy for a general case of $v=r / q \quad(1 / 2<v<1)$. As it is proven in Ref. [9] the electron-configuration with the minimum classical Coulomb energy is constructed by repeating the representative unit-configuration where $r$ electrons exist in sequential $q$ Landau orbitals. The number of empty orbitals per unit-configuration is $q-r$. All the empty orbitals are separated by one or more filled-orbitals at $1 / 2<v<1$. That is to say, all the empty orbitals are isolated as seen in Figures 2-5. Therefore the $q-r$ second-nearest pairs exist per unit-configuration due to the presence of the $q-r$ empty orbitals. The total numbers of the first and the second nearest pairs is equal to the total number of electrons as easily seen in Figures $2-5$. Therefore the number of nearest pairs becomes $r-(q-r)=2 r-q$ per unit-configuration. The total number of nearest electron pairs is equal to $(2 r-q)(N / r)$ and the total number of second nearest electron pairs is equal to $(q-r)(N / r)$ for the filling factor $v=r / q$. Consequently the total classical Coulomb energy is given by 


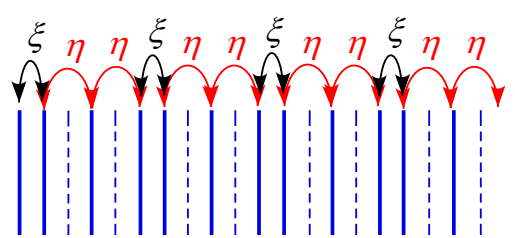

Figure 3. Electron configuration with the minimum classical Coulomb energy at $v=3 / 5$.

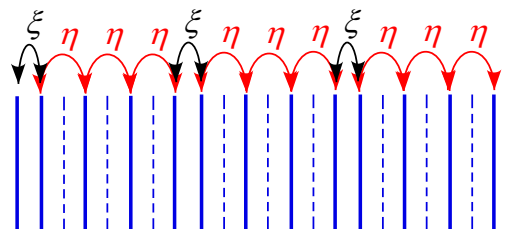

Figure 4. Electron configuration with the minimum classical Coulomb energy at $v=4 / 7$.

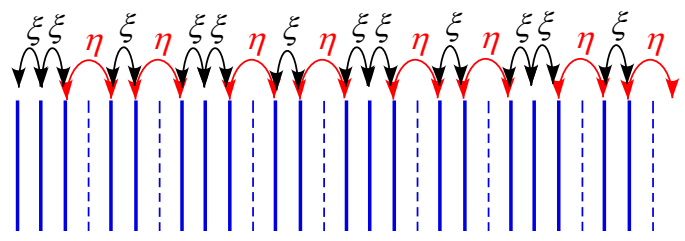

Figure 5. Electron configuration with the minimum classical Coulomb energy at $v=5 / 7$.

$$
\begin{aligned}
C\left(p_{1}, \cdots, p_{N}\right) & =[(\xi(2 r-q) / r)+(\eta(q-r) / r)] N+C_{\text {Macroscopic }}(\sigma) \\
& =[(2 \xi-\eta)-((\xi-\eta) q / r)] N+C_{\text {Macroscopic }}(\sigma) \text { for } v=r / q
\end{aligned}
$$

This equation is expressed by using $v$ as

$$
C\left(p_{1}, \cdots, p_{N}\right)=[(2 \xi-\eta)-(\xi-\eta) / v] N+C_{\text {Macroscopic }}(\sigma) \text { for } v=r / q
$$

From Equations (4) and (10) the expectation value of the total Hamiltonian is obtained as

$$
W\left(p_{1}, \cdots, p_{N}\right)=\sum_{i=1}^{N} E_{0}\left(p_{i}\right)+[(2 \xi-\eta)-(\xi-\eta) / v] N+C_{\text {Macroscopic }}(\sigma) \text { for } v=r / q
$$

The single electron eigenenergy $E_{0}\left(p_{i}\right)$ has been investigated in the previous papers and the result is the following form.

$$
E_{0}\left(p_{i}\right)=\lambda+U\left(\alpha_{i}\right)+\hbar e B /\left(2 m^{*}\right)
$$

where $\lambda$ expresses the ground state energy along the $\mathrm{z}$ direction (direction of the thickness in the thin conducting electron channel). Also $U\left(\alpha_{i}\right)$ is the potential along the y direction (Hall voltage direction). Substitution of Equation (12) into Equation (11) yields the expectation value of the total Hamiltonian as follows:

$$
W\left(p_{1}, \cdots, p_{N}\right)=\sum_{i=1}^{N}\left(\lambda+U\left(\alpha_{i}\right)+\hbar e B /\left(2 m^{*}\right)\right)+N(2 \xi-\eta)-N(\xi-\eta) / v+C_{\text {Macroscopic }}(\sigma)
$$

We put together the constant parts as follows;

$$
W\left(p_{1}, \cdots, p_{N}\right)=\left[f+\hbar e B /\left(2 m^{*}\right)-(\xi-\eta) / v\right] N+C_{\text {Macroscopic }}(\sigma)
$$

Therein $f$ is the constant value as 


$$
\begin{aligned}
& f=\lambda+\bar{U}+2 \xi-\eta \\
& \bar{U}=\sum_{i=1}^{N} U\left(\alpha_{i}\right) / N
\end{aligned}
$$

where $\bar{U}$ is the mean value of the potential along the $y$-direction. Equation (13b) gives the function-form of $W$, which is illustrated in Figure 6.

The function $W$ depends linearly upon $1 / v$ and the proportional coefficient $-(\xi-\eta) N$ is negative, because the classical Coulomb energy between the first nearest electron pair, $\xi$, is larger than that between the second nearest pair, $\eta$. Thus the expectation value of the total Hamiltonian $W$ changes continuously with $1 / v$ as in Figure 6. Accordingly the classical Coulomb energy has no energy-gap and so cannot produce the plateaus of Hall resistance. The confinement of the Hall resistance comes from another reason as studied in the previous papers [6] [9] [12] [13]. The allowed transitions of electron-pairs decrease abruptly when the filling factor $v$ deviates from the specific filling factor $v_{0}$. This structure is named "valley structure". Summation of the valley energy and $W$ gives the energy spectrum of the quasi 2D-electron system as examined in the next section.

\section{3. $v$-Dependence of the Total Energy}

We already calculated the energy of electron pairs placed in the nearest orbitals by employing the perturbation calculation in the previous papers [1]-[13]. The exact pair energy per electron is expressed by the following symbols $\chi(v)$ and $g(v)$ where $\chi(v)$ means the exact pair energy of the electrons placed in the nearest Landau orbitals and $g(v)$ means that of the more distant pairs. Then the total energy per electron $\varepsilon(v)$ is the sum of $\chi(v), g(v)$ and the expectation value $\gamma(v)=W / N$ as follows:

$$
\varepsilon(v)=\chi(v)+g(v)+\gamma(v)
$$

The exact pair energies are obtained by summing all orders of the perturbation energies as follows:

$$
\begin{aligned}
& \chi(v)=\sum_{n=2,3,4, \cdots} \chi_{n}(v) \\
& g(v)=\sum_{n=2,3,4, \cdots} g_{n}(v)
\end{aligned}
$$

Consequently the total energy $E_{\mathrm{T}}(v)$ of the quasi 2D electron system has been expressed as

$$
E_{\mathrm{T}}(v)=(\chi(v)+g(v)+\gamma(v)) N
$$

Equations (13b) and (14a, b) derive the following relation:

$$
\begin{gathered}
\gamma(v)=f+\hbar e B /\left(2 m^{*}\right)-(\xi-\eta) / v+\left(C_{\text {Macroscopic }}(\sigma) / N\right)=\hbar e B /\left(2 m^{*}\right)-a / v+b \\
a=(\xi-\eta), \quad b=\lambda+\bar{U}+2 \xi-\eta+\left(C_{\text {Macroscopic }} / N\right) \\
\bar{U}=\sum_{i=1}^{N} U\left(\alpha_{i}\right) / N
\end{gathered}
$$

Accordingly the total energy is a sum of the following five terms:

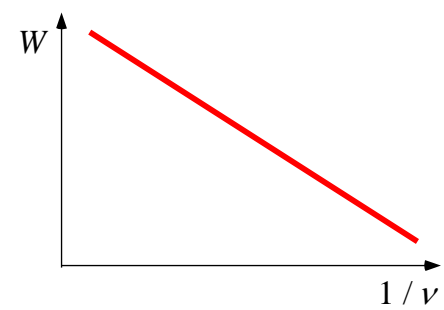

Figure 6. Expectation value $W$ of the total Hamiltonian. 


$$
E_{\mathrm{T}}(v)=\left(\chi(v)+g(v)+\left(e \hbar / 2 m^{*}\right) B-a / v+b\right) N
$$

Also the energy per electron is given by

$$
\varepsilon(v)=\chi(v)+g(v)+\left(e \hbar / 2 m^{*}\right) B-a / v+b
$$

If we change the gate voltage, then the value of the potential $\bar{U}$ varies. Accordingly the value of $b$ can be controlled by changing the gate voltage.

We examine the higher order perturbation energies. In the previous articles [9] [12] [13], we calculated the pair energy for a filling factor with an even number denominator. Therein all the quantum transitions from the nearest pairs are forbidden at the filling factors $v=(2 j-1) /(2 j)$ and $v=1 /(2 j)$. Therefore all order perturbation energies of the nearest electron (or hole) pair are zero;

$$
\chi_{n}(v)=0 \quad(n=2,3,4, \cdots) \text { for } v=(2 j-1) /(2 j) \text { and } v=1 /(2 j)
$$

which gives the exact pair energy of the electrons (or holes) placed in the nearest neighboring Landau orbitals as follows;

$$
\chi(v)=\sum_{n=2,3,4, \cdots} \chi_{n}(v)=0 \text { for } v=(2 j-1) /(2 j) \text { and } v=1 /(2 j)
$$

Next, we investigate the pair energy at the filling factor with an odd number denominator. The results are

$$
\begin{aligned}
& \chi(v)=-\sum_{n=2,3, \cdots} \frac{1}{2 j}\left(\frac{1}{2 j+1}\right)^{n-1}{ }^{n} Z \quad \text { at } v=2 j /(2 j+1) \\
& \chi(v)=-\sum_{n=2,3, \cdots} \frac{1}{j}\left(\frac{j-1}{2 j-1}\right)^{n-1}{ }^{n} Z \quad \text { at } v=j /(2 j-1) \\
& \chi(v)=-\sum_{n=2,3, \cdots}\left(\frac{1}{2 j+1}\right)^{n-1}{ }^{n} Z_{\mathrm{H}} \quad \text { at } v=1 /(2 j+1) \\
& \chi(v)=-\sum_{n=2,3, \cdots} \frac{1}{j}\left(\frac{j}{2 j+1}\right)^{n-1}{ }^{n} Z_{\mathrm{H}} \quad \text { at } v=j /(2 j+1)
\end{aligned}
$$

Equations 25(a)-(d) mean that the $n$-th order term has the multipliers $(1 /(2 j+1))^{n-1},((j-1) /(2 j-1))^{n-1}$, $(1 /(2 j+1))^{n-1}$ and $(j /(2 j+1))^{n-1}$, respectively. These multipliers become small for large $n$. We show several examples as follows;

$$
\begin{aligned}
& (1 /(2 j+1))^{n-1}=0.04,(j /(2 j+1))^{n-1}=0.16,((j-1) /(2 j-1))^{n-1}=0.11 \cdots \quad \text { for } n=3, j=2 \\
& (1 /(2 j+1))^{n-1}=0.008,(j /(2 j+1))^{n-1}=0.064,((j-1) /(2 j-1))^{n-1}=0.037 \cdots \quad \text { for } n=4, j=2
\end{aligned}
$$

The smallness of the multipliers means that the second order term is a main part of $\chi(v)$. We write again the second order term $\chi_{2}(v)$ for various filling factors:

$$
\begin{aligned}
& \chi_{2}(v)=-\frac{1}{2 j}\left(\frac{1}{2 j+1}\right) Z \quad \text { at } v=2 j /(2 j+1) \\
& \chi_{2}(v)=-\frac{1}{j}\left(\frac{j-1}{2 j-1}\right) Z \quad \text { at } v=j /(2 j-1) \\
& \chi_{2}(v)=-\left(\frac{1}{2 j+1}\right) Z_{\mathrm{H}} \quad \text { at } v=1 /(2 j+1) \\
& \chi_{2}(v)=-\frac{1}{j}\left(\frac{j}{2 j+1}\right) Z_{\mathrm{H}} \quad \text { at } v=j /(2 j+1)
\end{aligned}
$$




$$
\chi_{2}(v)=0 \text { at } v=(2 j-1) /(2 j) \text { and } v=1 /(2 j)
$$

We calculate the pair energies in the neighbourhood of $v=(j+1) /(2 j+1)$ and $v=j /(2 j+1)$. The results are

$$
\begin{aligned}
& \chi_{2}\left(v^{\prime}\right)=\frac{E_{\text {nearest pair }}}{N}=-\frac{((2 j-1) 2 s-2) s}{((2 j+1)(2 s)-2)} \frac{Z}{((j+1)(2 s)-1)} \quad \text { at } v^{\prime}=\frac{((j+1)(2 s)-1)}{((2 j+1)(2 s)-2)} \\
& \chi_{2}\left(v^{\prime}\right)=\frac{E_{\text {nearest pair }}}{N}=-\frac{((2 j-1) 2 s+2) s}{((2 j+1)(2 s)+2)} \frac{Z}{((j+1)(2 s)+1)} \quad \text { at } v^{\prime}=\frac{((j+1)(2 s)+1)}{((2 j+1)(2 s)+2)} \\
& \chi_{2}\left(v^{\prime}\right)=\frac{E_{\text {nearest pair }}}{N}=-\frac{((2 j-1) 2 s-2) s}{((2 j+1)(2 s)-2)} \frac{Z_{\mathrm{H}}}{((j)(2 s)-1)} \quad \text { at } v^{\prime}=\frac{((j)(2 s)-1)}{((2 j+1)(2 s)-2)} \\
& \chi_{2}\left(v^{\prime}\right)=\frac{E_{\text {nearest pair }}}{N}=-\frac{((2 j-1) 2 s+2) s}{((2 j+1)(2 s)+2)} \frac{Z_{\mathrm{H}}}{((j)(2 s)+1)} \quad \text { at } v^{\prime}=\frac{((j)(2 s)+1)}{((2 j+1)(2 s)+2)}
\end{aligned}
$$

For arbitrary fractional number $v$, we can calculate $\chi_{2}(v)$ by using the same procedure. When the denominator of the fractional number is large, the total number of the quantum transitions is calculated by a computer.

Because the higher order perturbation energy includes the small multipliers, $\varepsilon(v)$ is approximated by

$$
\varepsilon(v) \approx \chi_{2}(v)+g+\left(e \hbar / 2 m^{*}\right) B-a / v+b
$$

\section{Spectrum of the Total Energy versus Filling Factor}

The energy spectra are examined which is given by Equation (30). Therein the term $g$ indicates the non-nearest pair energy. Any non-nearest pair interleaves one or more Landau orbitals inside the pair. We have examined this effect in details in the article [12]. The energies of the non-nearest pairs are smaller than that of the nearest pairs for $v<2$. Accordingly we may ignore the $v$-dependence of $g(v)$ in Equation (30). We draw four graphs of $\varepsilon(v)$ in the neighbourhood of $v=2 / 3,1 / 2,2 / 5,4 / 7$ in Figure 7.
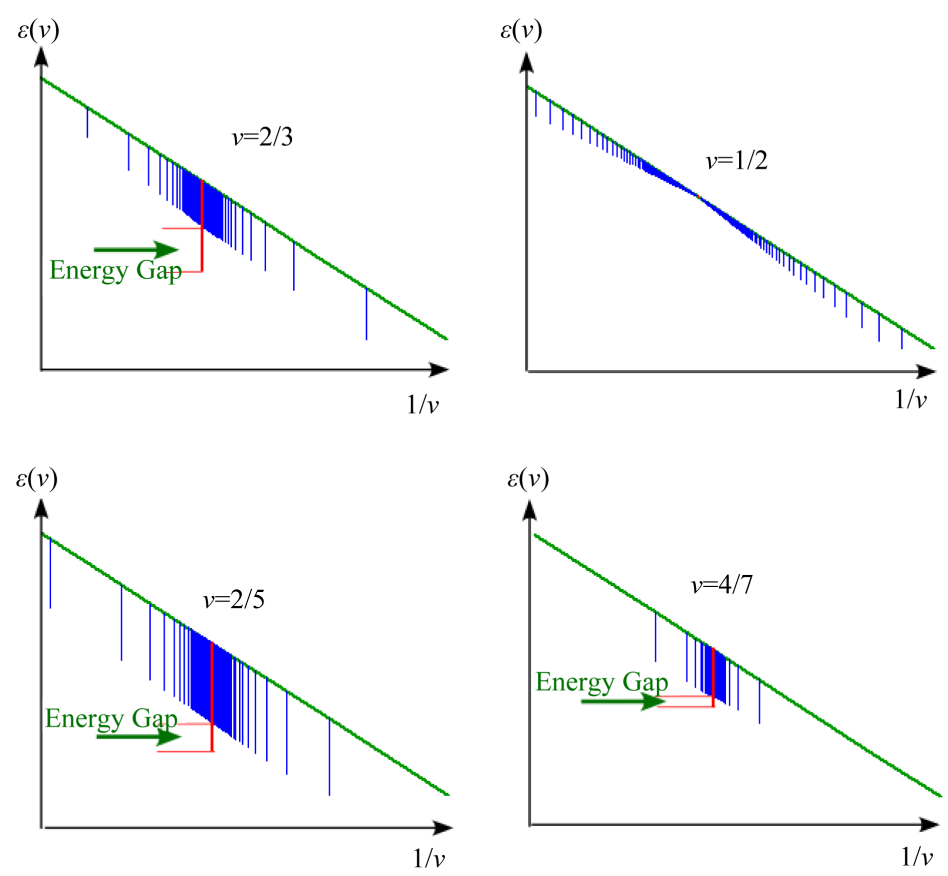

Figure 7. Energy spectra in the neighbourhood of $v=2 / 3,1 / 2,2 / 5,4 / 7$. 
In Figure 7, dark green lines indicate the function $Q(v)$ defined by

$$
Q(v)=g+(e \hbar / 2 m) B-a / v+b
$$

We illustrate the value of $\chi_{2}(v)$ by vertical bars in Figure 7, where the lengths of the bars are the absolute values of $\chi_{2}(v)$. The upper end of each vertical bar is placed on the dark green line namely $Q(v)$. Because $\chi_{2}(v)$ is negative, the lower ends of each vertical bars indicate the values of $\varepsilon(v)$ :

$$
\varepsilon(v) \approx \chi_{2}(v)+Q(v)
$$

Red vertical bars express $\chi_{2}(v)$ at $v=2 / 3,2 / 5$ and 4/7. Blue vertical bars indicate $\chi_{2}(v)$ in the neighbourhood of $v=1 / 2,2 / 3,2 / 5$ and 4/7 which is given by Equations 29(a)-(d). The energy spectra in Figure 7 have the following two features:

1) The lower end of the red bar in respective figure is lower than that of the blue bars in the neighbourhood of $v=2 / 3,2 / 5,4 / 7$. Then the energy spectra yield a valley, the depth of which is shown by energy gap shown in Figure 7.

2) There is no valley at $v=1 / 2$. The blue vertical bar approaches the base line (dark green line) near $v=1 / 2$.

The denominators of the filling factors in Equations 29(a)-(d) are even numbers. Just to be sure, we calculate the energy $\chi_{2}(v)$ for the nearest pairs at $v=(4 s+1) /(6 s+1)$ and $v=(4 s-1) /(6 s-1)$ with even number denominators. The nearest pair energies are given by

$$
\begin{aligned}
& E_{\text {nearest pair }}=-\frac{2 s^{2}}{(4 s+1)(6 s+1)} Z N \quad \text { for } v=(4 s+1) /(6 s+1) \\
& E_{\text {nearest pair }}=-\frac{2 s^{2}}{(4 s-1)(6 s-1)} Z N \quad \text { for } v=(4 s-1) /(6 s-1)
\end{aligned}
$$

The energies per electron are

$$
\begin{array}{ll}
\chi_{2}(v)=-\frac{2 s^{2}}{(4 s+1)(6 s+1)} Z \quad \text { for } v=(4 s+1) /(6 s+1) \\
\chi_{2}(v)=-\frac{2 s^{2}}{(4 s-1)(6 s-1)} Z \quad \text { for } v=(4 s-1) /(6 s-1)
\end{array}
$$

These pair energies are shown in Figure 8 together with the energies of Figure 7. The green vertical bars indicate $\chi_{2}(v)$ at $v=(4 s+1) /(6 s+1)$ and $v=(4 s-1) /(6 s-1)$ with odd number denominators. The blue bars indicate $\chi_{2}(v)$ at $v=(4 s-1) /(6 s-2)$ and $v=(4 s+1) /(6 s+2)$ with even number denominators.

The green vertical bars are drawn after showing the blue bars in the Mathematica program. Only green bars are seen in the vicinity of $v=2 / 3$ because the blue bars are hidden by the dense green bars. We can see both blue and green bars when we move from $v=2 / 3$. The lengths of the green and blue bars are almost the same.

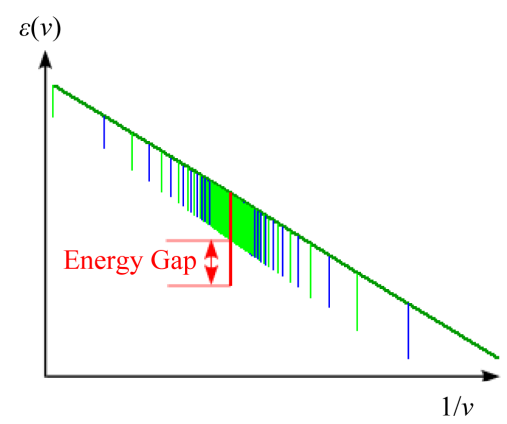

Figure 8. Energy spectrum in the neighbourhood of $v=2 / 3$. 
The limiting value, $\lim _{v \rightarrow 2 / 3} \chi_{2}(v)$, for the filling factors with odd number denominators is the same as that with even number denominators. So the difference of $\chi_{2}(v)$ between even and odd number denominators is negligibly small in the neighbourhood of $v=2 / 3$.

The energy spectra in Figure 7 and Figure 8 are drawn separately in the neighbourhood of the four filling factors. Some readers may want to know the spectrum in a wider region of the filling factor. We show it in Figure 9. The $v$ dependence of $\varepsilon(v)$ (see Equation (30)) is induced by only two parameters $Z$ and $a$ which depend upon the size, thickness and shape of a quantum Hall device. (Note 1: $\chi_{2}(v)$ is expressed by $Z$. Note 2: The other parameters $b, g, \cdots$ don't yield $v$-dependence.) We draw the energy spectrum in Figure 9 for the parameter ratio $Z / a=0.5$ as an example.

We find many ranges of absent vertical-bar in Figures 7-9, because the present author doesn't calculate the value of $\chi_{2}(v)$ yet in these ranges. Of course we can calculate $\chi_{2}(v)$ inside the ranges by using a computer and get the more dense vertical bars.

Hitherto, a few theorists have calculated the energy spectrum of FQH states. As an example Halperin's result [15] is shown in Figure 10 which has many cusps in the energy spectrum. The function-shape of Halperin's result is quite different from that of the present theory. Our theoretical spectrum-form is important to yield the Hall resistance curve in many experimental data as will be clarified in the next sections.

\section{Behaviour of Hall Resistance Curve Near $v=1 / 2,3 / 4,1 / 4$ And So On}

We draw three graphs of $\varepsilon(v)$ in the neighbourhood of $v=1 / 2$ in Figure 11. The left panel shows the energy

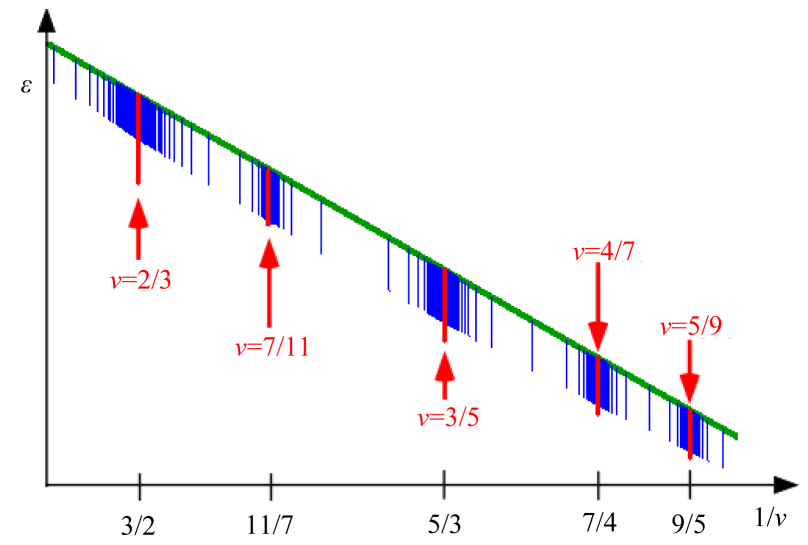

Figure 9. Energy spectrum of the present theory.

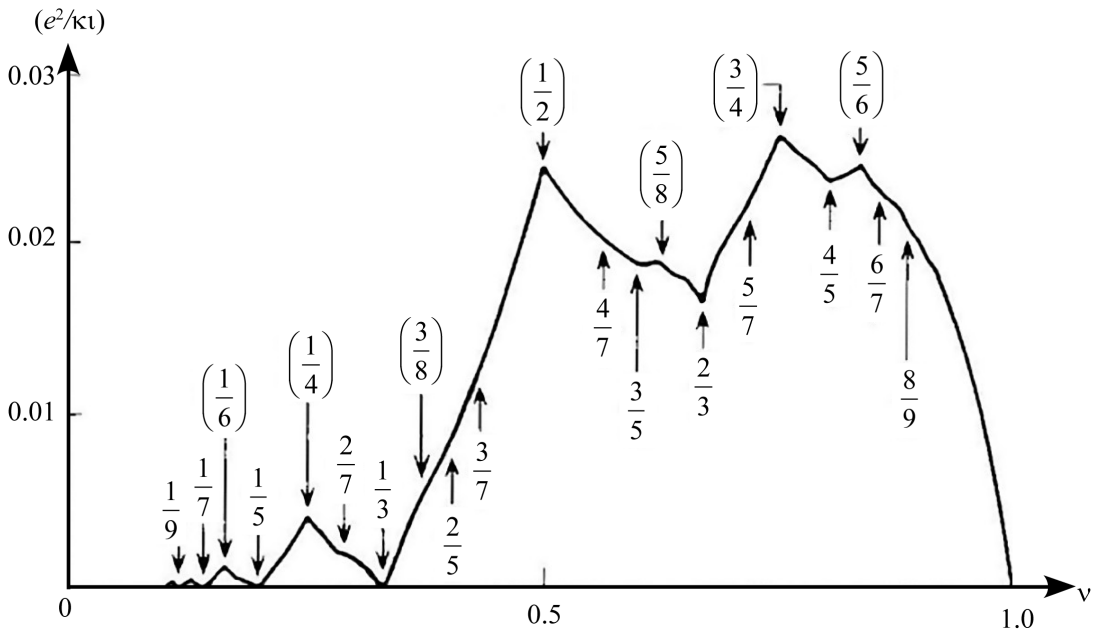

Figure 10. Energy spectrum of Halperin's result [15]. 


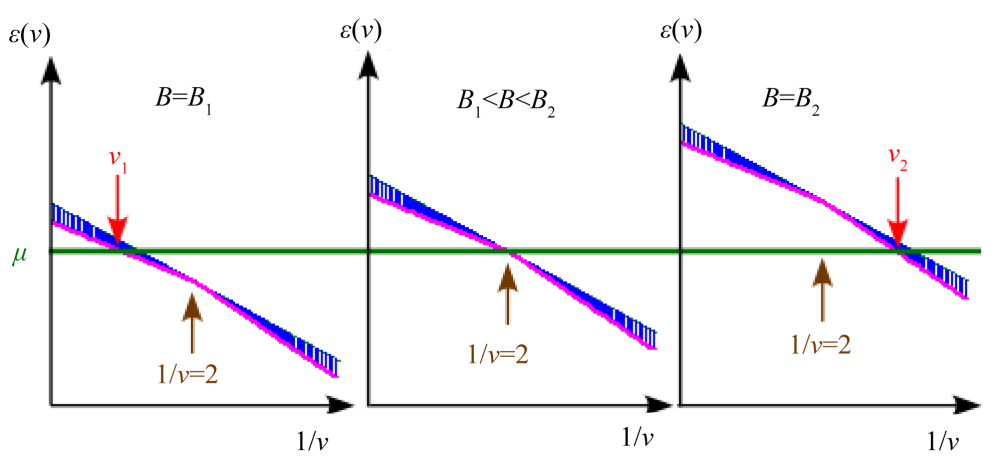

Figure 11. Magnetic field dependence of Energy spectrum near $v=1 / 2$.

spectrum at the magnetic field $B=B_{1}$, the middle one $B_{1}<B<B_{2}$ and the right one $B=B_{2}$. The energy $\varepsilon(v)$ increases with increment of $B$ because of the term $\left(e \hbar / 2 m^{*}\right) B$ in Equation (30). We draw the chemical potential $\mu$ by the dark green line in Figure 11. The pink curve shows the lower end of each blue bar which indicates the value of $\varepsilon(v)$.

The Fermi-Dirac distribution function $n(\varepsilon)$ is given by

$$
n(\varepsilon)=\frac{1}{\exp \left((\varepsilon-\mu) / k_{\mathrm{B}} T\right)+1}
$$

where $k_{\mathrm{B}}$ is the Boltzmann's constant and $T$ is the temperature. At a low temperature all the states with $\varepsilon<\mu$ are occupied by electrons and the states with $\varepsilon>\mu$ are empty.

The left panel of Figure 11 shows the energy spectrum at the magnetic field $B=B_{1}$ where $\varepsilon(v)$ is higher than $\mu$ for any filling factor $v>v_{1}$ and $\varepsilon(v)$ is lower than $\mu$ for any filling factor $v<v_{1}$. (Note that the horizontal axis indicates $1 / v$ and so the filling factor in the left is larger than one in the right.) Accordingly all the electron-states of $v<v_{1}$ are filled with electron, and any state of $v>v_{1}$ is empty at low temperatures. That is to say, the filling factor becomes $v_{1}$ at $B=B_{1}$. Also the filling factor becomes $v_{2}$ at $B=B_{2}$ as seen in the right panel of Figure 11.

Next, we examine in the range of $B_{1}<B<B_{2}$ how the inverse of the filling factor $1 / v$ depends upon the magnetic field $B$ under the fixed value of the chemical potential $\mu$. When the filling factor is nearly equal to 1/2, the nearest pair energy $\chi_{2}(v)$ is very small as in Figure 11. Then $\varepsilon(v)$ is almost equal to $Q(v)$ due to Equation (32). So the value of the filling factor is determined by the relation $Q(v) \approx \mu$ :

$$
\begin{aligned}
& \varepsilon(v) \approx Q(v)=g+(e \hbar / 2 m) B-a / v+b \approx \mu \text { near } v=1 / 2 \\
& a / v \approx g+\left(e \hbar / 2 m^{*}\right) B+b-\mu \\
& 1 / v \approx\left(e \hbar /\left(2 m^{*} a\right)\right) B+(b+g-\mu) / a \text { near } v=1 / 2
\end{aligned}
$$

Equation (36) means that the inverse of the filling factor, $1 / v$, is linearly dependent upon the magnetic field in the neighbourhood of $v=1 / 2$. We next study the Hall resistance. As an example we consider the ground state with $v=1 / 2$ which is composed of the electron configuration repeating of the unit (filled empty). The empty states yield no electric current. So, the electric current is $1 / 2$ times of the current in the integer quantum Hall state with $v=1$. This means that the Hall resistance of the present theory is given by

$$
R_{\mathrm{H}}=\frac{\left(V_{2}-V_{1}\right)}{I}=\frac{2 \pi \hbar}{e^{2}(1 / 2)} \text { for } v=1 / 2
$$

For arbitrary filling factor $v$, the Hall resistance is equal to

$$
R_{\mathrm{H}}=\frac{2 \pi \hbar}{e^{2} v} \text { for any filling factor } v
$$


Substitution of Equation (36) into Equation (38) yields the following relation:

$$
R_{\mathrm{H}} \approx \frac{2 \pi \hbar}{e^{2}}\left(\left(e \hbar / 2 m^{*} a\right) B+(b+g-\mu) / a\right) \text { near } v=1 / 2
$$

This theoretical result shows that the Hall resistance depends linearly upon the magnetic field in the neighbourhood of $v=1 / 2$. One of the experimental data is shown in Figure 12.

The experimental value of the Hall resistance depends linearly upon the magnetic field near $v=1 / 2$ as easily seen on the upper panel. Equation (39) means that the theoretical result is in good agreement with the experimental data.

We next examine the diagonal resistance. The states near $v=1 / 2$ have no valley structure and so the excitation energy is nearly equal to zero. Accordingly the quantum transitions occur easily by electron scatterings. Consequently the diagonal resistance $R_{\mathrm{xx}}$ is predicted to be finite and almost constant in the neighbourhood of $v=1 / 2$. This property appears in the experimental data as in the lower panel of Figure 12 .

We discuss the case with the peak structure. For an example $v=3 / 4$, the probability of the $v=3 / 4$ state is smaller than the probabilities with $v=(3 / 4) \pm \varepsilon$ because of the energy peak structure. Accordingly the state with $v=(3 / 4)+\varepsilon$ varies to the state with $v=(3 / 4)-\varepsilon$ skipping the $v=3 / 4$ state ( $\varepsilon$ is an infinitesimally small number) when the magnetic field is increased. Then the Hall resistance is linearly dependent on the magnetic field in the neighbourhood of $v=3 / 4$. Thus the FQH state near the peak structure has a property similar to one in the neighbourhood of $v=1 / 2$.

\section{Explanation for Appearance of Plateaus in the Hall Resistance Curve}

Many Hall plateaus have been observed at various fractional filling factors in the experiments [16]-[25]. We examine how the theoretical function-form of the energy $\varepsilon(v)$ produces the plateaus in the Hall resistance curve. We draw three figures of $\varepsilon(v)$ in the neighbourhood of $v=2 / 3$ for each of magnetic fields, $B=B_{3}$, $B_{3}<B<B_{4}$ and $B=B_{4}$, respectively in Figure 13 .

When the magnetic field becomes stronger, the value of $\varepsilon(v)$ becomes higher because of $\left(e \hbar / 2 m^{*}\right) B$ in Equation (30). We show the chemical potential $\mu$ by the green line. The pink curve indicates the value of $\varepsilon(v)$ in the neighbourhood of $v=2 / 3$ except $v=2 / 3$. The valley appearing at $v=2 / 3$ is shown in red bar.

We can find that the energy $\varepsilon(2 / 3)$ is lower than the chemical potential $\mu$ in the range of $B_{3}<B<B_{4}$ as in Figure 13. Therefore, the state with $v=2 / 3$ is filled with electrons at low temperatures. By contrast, $\varepsilon(v>2 / 3)$ is higher than the chemical potential $\mu$ in the range of $B_{3}<B<B_{4}$ and so all the states with $v>2 / 3$ are empty at low temperatures. Consequently, the filling factor is confined to $2 / 3$ in the range of $B_{3}<B<B_{4}$ [17] [18]. Then, the Hall resistance is given as follows;

$$
R_{\mathrm{H}}=\frac{\left(V_{2}-V_{1}\right)}{I}=\frac{2 \pi \hbar}{v e^{2}}=\frac{3}{2} \times \frac{2 \pi \hbar}{e^{2}} \text { in the range of } B_{3}<B<B_{4}
$$

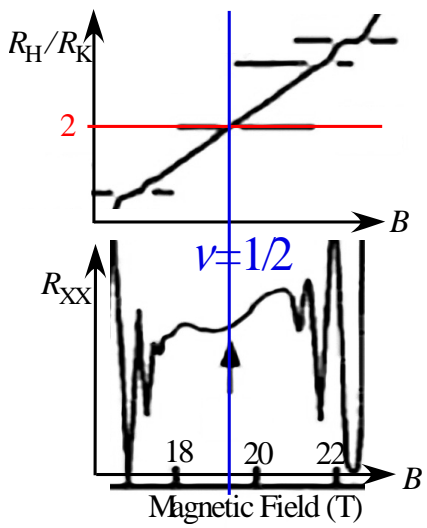

Figure 12. Experimental results of Hall resistance $R_{\mathrm{H}}$ and diagonal resistance $R_{\mathrm{xx}}$ [16]. $R_{\mathrm{K}}$ is the von Klitzing constant. 


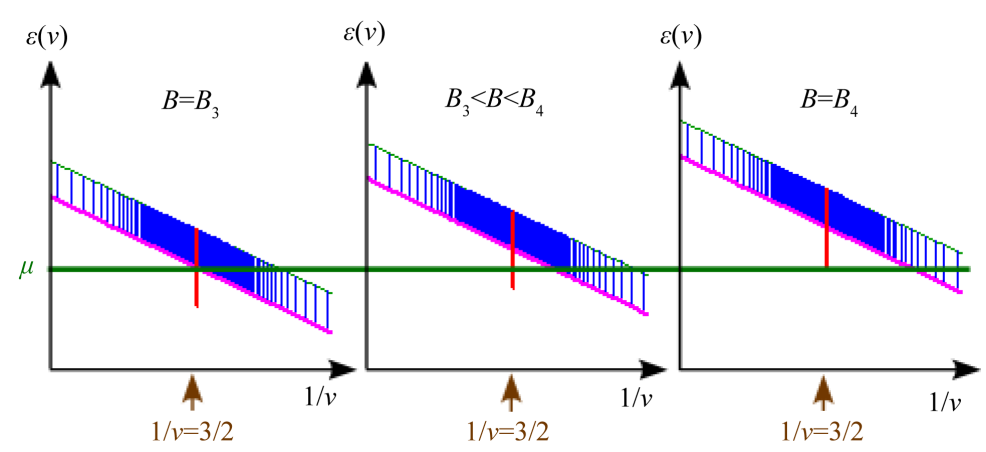

Figure 13. Magnetic field dependence of Energy spectrum near $v=2 / 3$.

That is to say, the Hall resistance $R_{\mathrm{H}}$ takes a constant value $(3 / 2) \times 2 \pi \hbar / e^{2}$ in the range of $B_{3}<B<B_{4}$. We next examine two more examples $v=3 / 5$ and $4 / 5$. The two states with $v=3 / 5$ and $4 / 5$ have also large valleys in their energy spectra. The experimental data are shown in Figure 14 where three plateaus appear at $v=$ $2 / 3,3 / 5$ and $4 / 5$. Thus the present theoretical results are in good agreement with the experimental data. We theoretically conclude that the appearance of the plateaus in the Hall resistance curve originates from the valley structure.

The ground state with $v=2 / 3$ has the excitation-energy (1/3) $Z$ because the electron pair is destroyed by the energy $2 \times\left|\chi_{2}(2 / 3)\right|$ of two electrons. So the ground state with $v=2 / 3$ becomes stable at low temperatures. Because the excitation energy is very large, the electron scatterings are suppressed. The absence of the scatterings yields vanishing of the diagonal resistance $R_{x x}$ in the width $\Delta B$ of Figure 15. Thus the vanishing width of diagonal resistance $\Delta B$ is equal to the width of Hall plateau, $B_{4}-B_{3}$ with $v=2 / 3$.

The energy depth of the valley is related to the width $\Delta B=B_{4}-B_{3}$ as seen below. The left panel of Figure 13 shows that the limiting value $\lim _{v \rightarrow 2 / 3+0} \varepsilon(v)$ at $B=B_{3}$ is equal to the chemical potential $\mu$ as;

$$
\lim _{v \rightarrow 2 / 3+0} \varepsilon\left(v, B=B_{3}\right)=\lim _{v \rightarrow 2 / 3+0}(\chi(v))+g(2 / 3)+\left(e \hbar / 2 m^{*}\right) B_{3}-a /(2 / 3)+b \approx \mu
$$

The right panel of Figure 13 gives the following equation:

$$
\varepsilon\left(v=2 / 3, B=B_{4}\right)=\chi(2 / 3)+g(2 / 3)+\left(e \hbar / 2 m^{*}\right) B_{4}-a /(2 / 3)+b \approx \mu
$$

Subtraction of Equation (42) from Equation (41) yields the following relation:

$$
\left[\lim _{v \rightarrow 2 / 3+0} \chi(v)\right]-\chi(2 / 3)+\left(e \hbar / 2 m^{*}\right)\left(B_{3}-B_{4}\right)=0
$$

So the energy gap (depth of the valley) in Figure 7 and Figure 8 is equal to

$$
\text { energy gap (depth of the valley) }=\left[\lim _{v \rightarrow 2 / 3+0} \chi(v)\right]-\chi(2 / 3)
$$

Substitution of Equation (44) into Equation (43) gives the relation as

$$
\text { energy gap (depth of the valley) }=\hbar e\left(B_{4}-B_{3}\right) /\left(2 m^{*}\right)=\hbar e \Delta B /\left(2 m^{*}\right) \text {. }
$$

Thus the depth of the valley yields the experimental width $\Delta B=B_{4}-B_{3}$.

\section{Comparison of the Present Theory with Experimental Data}

We have found the valley structure at $v=2 / 3,1 / 3,4 / 5,3 / 5,2 / 5,1 / 5,4 / 7,3 / 7 \cdots$, the flat structure at $v=1 / 2$ and the peak structure at $3 / 4,1 / 4, \cdots$. The flat and peak structures produce a linear dependence of the Hall resistance versus the magnetic field as clarified in Section 4. The valley structure produces a confinement of Hall resistance because only one FQH state is realized in some ranges of the magnetic field at low temperatures.

We compare the depths of the valleys with the experimental data of the diagonal resistance [17] [18]. If an experiment is done using an ideal device without impurity and lattice defect at zero temperature, then $R_{\mathrm{Xx}}$ is 


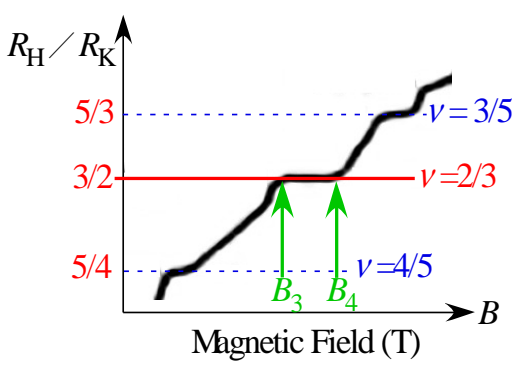

Figure 14. Experimental data [17] of Hall resistance $R_{\mathrm{H}}$ near $v=4 / 5,2 / 3,3 / 5$.

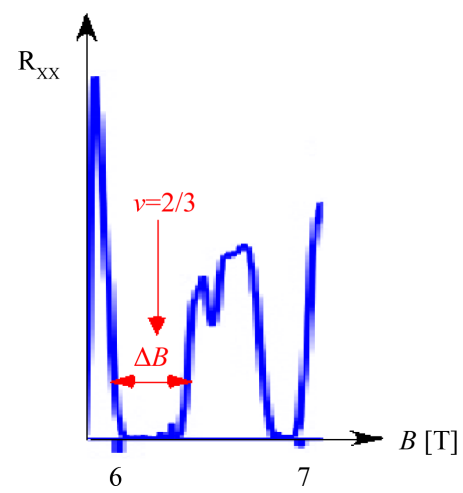

Figure 15. Experimental data of diagonal resistance $R_{x x}$ near $v=2 / 3$ in [17].

zero in several ranges of magnetic field. But the actual experiments employ devices with impurities and lattice defects and are carried out at a finite temperature. Therefore, the diagonal resistance is very small but not zero. So, we roughly estimate the width $W(v)$ from the experimental data as follows; we take the width where $R_{\mathrm{xx}}$ is lower than the green line in Figure 16.

Then, the experimental widths are

$$
\begin{aligned}
& W(2 / 3) \approx 0.35[\mathrm{~T}], W(3 / 5) \approx 0.16[\mathrm{~T}], W(4 / 7) \approx 0.09[\mathrm{~T}], \\
& W(4 / 9) \approx 0.07[\mathrm{~T}], W(3 / 7) \approx 0.12[\mathrm{~T}], \\
& W(2 / 5) \approx 0.19[\mathrm{~T}], W(1 / 3) \approx 0.9[\mathrm{~T}]
\end{aligned}
$$

Our second order calculations give the energy gaps (depths of the valleys) as;

$$
\begin{aligned}
& \Delta \varepsilon_{ \pm}(2 / 3)=-(1 / 12) Z, \Delta \varepsilon_{ \pm}(3 / 5)=-(1 / 30) Z, \Delta \varepsilon_{ \pm}(4 / 7)=-(1 / 56) Z, \\
& \Delta \varepsilon_{ \pm}(4 / 9)=-(1 / 72) Z, \Delta \varepsilon_{ \pm}(3 / 7)=-(1 / 42) Z_{\mathrm{H}}, \\
& \Delta \varepsilon_{ \pm}(2 / 5)=-(1 / 20) Z_{\mathrm{H}}, \Delta \varepsilon_{ \pm}(1 / 3)=-(1 / 6) Z_{\mathrm{H}}
\end{aligned}
$$

Neglecting the magnetic field dependence of $Z$ and using the approximate relation $Z \approx Z_{\mathrm{H}}$, Equations (47) gives the theoretical ratio of the energy gaps (depths of the valleys) as follows:

$$
\text { theoretical ratio }=\frac{1}{12}: \frac{1}{30}: \frac{1}{56}: \frac{1}{72}: \frac{1}{42}: \frac{1}{20}: \frac{1}{6} \approx 0.35: 0.14: 0.08: 0.06: 0.1: 0.21: 0.7
$$

The ratio of the experimental values is derived from Equations (46) as

$$
\begin{aligned}
\text { experimental ratio } & =W\left(\frac{2}{3}\right): W\left(\frac{3}{5}\right): W\left(\frac{4}{7}\right): W\left(\frac{4}{9}\right): W\left(\frac{3}{7}\right): W\left(\frac{2}{5}\right): W\left(\frac{1}{3}\right) \\
& \approx 0.35: 0.16: 0.09: 0.07: 0.12: 0.19: 0.9
\end{aligned}
$$




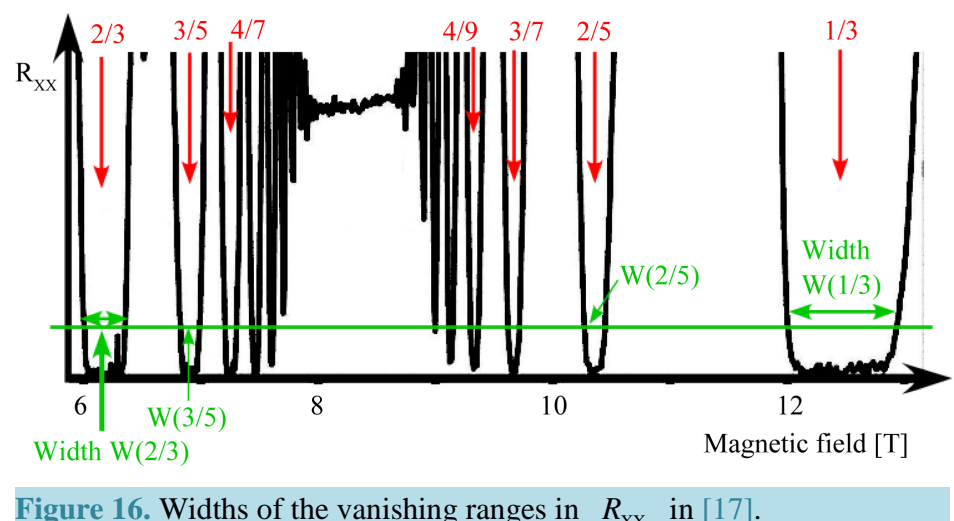

Thus, the theoretical result, namely ratio (48), is in good agreement with the experimental result (49) in spite of our rough estimation.

\section{Discussion and Summary}

There are two types of the traditional theories in the investigations of FQHE: one employs the quasi particle with a fractional charge [15] [26]-[29] and the other employs the quasi particle which is an electron binding to an even number of flax quanta namely composite fermion [30]-[41]. The original Hamiltonian is described by the normal electrons and therefore the quasi-particles should be expressed by the wave function of many electrons. The wave function is unknown. The other problems are discussed in the Appendix. The present article has investigated the FQHE on the basis of the fundamental Hamiltonian without any quasi particle. When the filling factor deviates from the specific fractional numbers, the allowed quantum transitions decrease abruptly by the combined effect of the three properties which are the Fermi-Dirac statistics, the most uniform configuration of electrons and the momentum conservation along the $x$-axis. So the pair energy takes a minimum value at the specific filling factors discontinuously. Thus, the theoretical energy spectrum has the valley structure which yields the Hall resistance confinements. The theoretical results are in good agreement with the experimental data.

\section{Acknowledgements}

The author expresses his heartfelt appreciation for the encouragement of Professor Koichi Katsumata, Professor Masayuki Hagiwara, Professor Hidenobu Hori, Professor Yasuyuki Kitano and Professor Takeji Kebukawa. I cannot complete this article without their support.

\section{References}

[1] Sasaki, S. (2000) Binding Energy, Polarization of Fractional Quantum Hall State. Proceedings of the 25th International Conference on the Physics of Semiconductors, Part II, Osaka, 17-22 September 2000, Springer, 925-926.

[2] Sasaki, S. (2000) Physica B: Condensed Matter, 281-282, 838-839. http://dx.doi.org/10.1016/S0921-4526(99)00840-6

[3] Sasaki, S. (2003) Surface Science, 532-535, 567-575. http://dx.doi.org/10.1016/S0039-6028(03)00091-8

[4] Sasaki, S. (2004) Surface Science, 566-568, 1040-1046. http://dx.doi.org/10.1016/j.susc.2004.06.101

[5] Sasaki, S. (2005) Binding Energies and Spin Polarizations of Fractional Quantum Hall States, In: Norris, C.P., Ed., Surface Science: New Research, Nova Science Publishers, New York, 103-161.

[6] Sasaki, S. (2008) Journal of Physics, 100, Article ID: 042021. http://dx.doi.org/10.1088/1742-6596/100/4/042021

[7] Sasaki, S. (2008) Journal of Physics, 100, Article ID: 042022. http://dx.doi.org/10.1088/1742-6596/100/4/042022

[8] Sasaki, S. (2010) Journal of Surface Science and Nanotechnology, 8, 121-124. http://dx.doi.org/10.1380/ejssnt.2010.121

[9] Sasaki, S. (2012) Advances in Condensed Matter Physics, 2012, Article ID: 281371. http://dx.doi.org/10.1155/2012/281371

[10] Sasaki, S. (2013) Journal of Modern Physics, 4, 1-7. http://dx.doi.org/10.4236/jmp.2013.49A001

[11] Sasaki, S. (2013) ISRN Condensed Matter Physics, 2013, Article ID: 489519. http://dx.doi.org/10.1155/2013/489519 
[12] Sasaki, S. (2014) ISRN Condensed Matter Physics, 2014, Article ID: 468130. http://dx.doi.org/10.1155/2014/468130

[13] Sasaki, S. (2015) Journal of Modern Physics, 6, 584-600. http://dx.doi.org/10.4236/jmp.2015.65064

[14] Sasaki, S. (2015) Journal of Modern Physics, 6, 794-810. http://dx.doi.org/10.4236/jmp.2015.66085

[15] Halperin, B.I. (1984) Physical Review Letters, 52, 1583-1586. http://dx.doi.org/10.1103/PhysRevLett.52.1583

[16] Stormer, H.L. (1999) Reviews of Modern Physics, 71, 875-889. http://dx.doi.org/10.1103/RevModPhys.71.875

[17] Pan, W., Stormer, H.L., Tsui, D.C., Pfeiffer, L.N., Baldwin, K.W. and West, K.W. (2002) Physical Review Letters, 88, Article ID: 176802. http://dx.doi.org/10.1103/PhysRevLett.88.176802

[18] Pan, W., Stormer, H.L., Tsui, D.C., Pfeiffer, L.N., Baldwin, K.W. and West, K.W. (2003) Physical Review Letters, 90, Article ID: 016801. http://dx.doi.org/10.1103/PhysRevLett.90.016801

[19] Tsui, D.C. and Gossard, A.C. (1981) Applied Physics Letters, 38, 550. http://dx.doi.org/10.1063/1.92408

[20] Tsui, D.C., Störmer, H.L. and Gossard, A.C. (1982) Physical Review B, 25, 1405-1407. http://dx.doi.org/10.1103/PhysRevB.25.1405

[21] Tsui, D.C., Stormer, H.L. and Gossard, A.C. (1982) Physical Review Letters, 48, 1559-1562. http://dx.doi.org/10.1103/PhysRevLett.48.1559

[22] Willet, R., Eisenstein, J.P., Stormer, H.L., Tsui, D.C., Gossard, A.C. and English, J.H. (1987) Physical Review Letters, 59, 1776-1779. http://dx.doi.org/10.1103/PhysRevLett.59.1776

[23] Eisenstein, J.P. and Stormer, H.L. (1990) Science, 248, 1510-1516. http://dx.doi.org/10.1126/science.248.4962.1510

[24] Stormer, H.L. (2002) Nobel Lectures, Physics 1996-2000. World Scientific, Singapore, 295-325.

[25] Novoselov, K.S., Jiang, Z., Zhang, Y., Morozov, S.V., Stormer, H.L., Zeitler, U., Maan, J.C., Boebinger, G.S., Kim, P. and Geim, A.K. (2007) Science, 315, 1379. http://dx.doi.org/10.1126/science.1137201

[26] Laughlin, R.B. (1983) Physical Review B, 27, 3383-3389. http://dx.doi.org/10.1103/PhysRevB.27.3383

[27] Laughlin, R.B. (1983) Physical Review Letters, 50, 1395-1398. http://dx.doi.org/10.1103/PhysRevLett.50.1395

[28] Haldane, F.D.M. (1983) Physical Review Letters, 51, 605-608. http://dx.doi.org/10.1103/PhysRevLett.51.605

[29] Girvin, S.M. (1984) Physical Review B, 29, 6012-6014. http://dx.doi.org/10.1103/PhysRevB.29.6012

[30] Jain, J.K. (2014) Indian Journal of Physics, 88, 915-929. http://dx.doi.org/10.1007/s12648-014-0491-9

[31] Jain, J.K. (2007) Composite Fermions. Cambridge University Press., New York. http://dx.doi.org/10.1017/CBO9780511607561

[32] Das Sarma, S. (1996) Localization, Metal-Insulator Transitions, and Quantum Hall Effect. In: Das Sarma, S. and Pinczuk, A., Eds., Perspectives in Quantum Hall Effects: Novel Quantum Liquids in Low-Dimensional Semiconductor Structures, Wiley, New York, 1-36. http://dx.doi.org/10.1002/9783527617258.ch1

[33] Jain, J.K. and Kamilla, R.K. (1998) Composite Fermions: Particles of the Lowest Landau Level. In: Heinonen, O., Ed., Composite Fermions: A Unified View of the Quantum Hall Regime, World Scientific, New York, 1-90. http://dx.doi.org/10.1142/9789812815989_0001

[34] Jain, J.K. (1989) Physical Review Letters, 63, 199-202. http://dx.doi.org/10.1103/PhysRevLett.63.199

[35] Kamilla, R.K., Wu, X.G. and Jain, J.K. (1996) Physical Review Letters, 76, 1332-1335. http://dx.doi.org/10.1103/PhysRevLett.76.1332

[36] Jain, J.K. and Kamilla, R.K. (1997) Physical Review B, 55, R4895-R4898. http://dx.doi.org/10.1103/PhysRevB.55.R4895

[37] Park, K. and Jain, J.K. (1998) Physical Review Letters, 80, 4237-4240. http://dx.doi.org/10.1103/PhysRevLett.80.4237

[38] Jain, J.K. (2000) Physics Today, 53, 39-45. http://dx.doi.org/10.1063/1.883035

[39] Halperin, B.I. (2003) Physica E: Low-Dimensional Systems and Nanostructures, 20, 71-78. http://dx.doi.org/10.1016/j.physe.2003.09.022

[40] Murthy, G. and Shankar, R. (2003) Reviews of Modern Physics, 75, 1101-1158. http://dx.doi.org/10.1103/RevModPhys.75.1101

[41] Sitko, P., Yi, K.-S. and Quinn, J.J. (1997) Physical Review B, 56, 12417-12421. http://dx.doi.org/10.1103/PhysRevB.56.12417 


\section{Appendix}

The present author has some questions;

1) If the charge of the quasi particle is $v e$, then the quantum Hall resistance is confined to $R_{\mathrm{H}}=2 \pi \hbar /(e v)^{2}$ (not $2 \pi \hbar /\left(e^{2} v\right)$ ).

2) The external magnetic field is applied to the quasi 2D-electron system. The strength of the field can be varied continuously. So the electrons move in a magnetic field with continuous strength. The quasi 2D-electron system has no special boundary as in a superconducting ring. So, the flux quantization cannot be derived from the fundamental Hamiltonian. Also we cannot lead the binding energy between an electron and flux quanta from the Hamiltonian. 